\title{
Les budgets globaux, une fausse bonne idée?
}

\section{Bruno Kesseli}

Dr méd. et lic. phil, rédacteur en chef

\author{
Les budgets globaux sont de plus en plus discutés sur le plan national. Dans ce \\ contexte, la FMH et d'autres acteurs de la santé ${ }^{1}$ ont organisé à Berne le séminaire \\ «Les répercussions des budgets globaux sur les patients - regards sur l'étranger, \\ expériences suisses et aspects juridiques».
}

La manifestation avait pour objectif de permettre aux experts de la santé qui travaillent sur le sujet de se faire une image réaliste des avantages et inconvénients des budgets globaux dans le domaine de la santé. C'est pourquoi, comme l'a souligné dans son discours d'ouverture Willy Oggier, économiste de la santé et animateur du séminaire, l'accent a été mis sur l'expérience pratique des orateurs venus d'Allemagne et de Suisse. Tous ont largement répondu à cette attente. Le premier

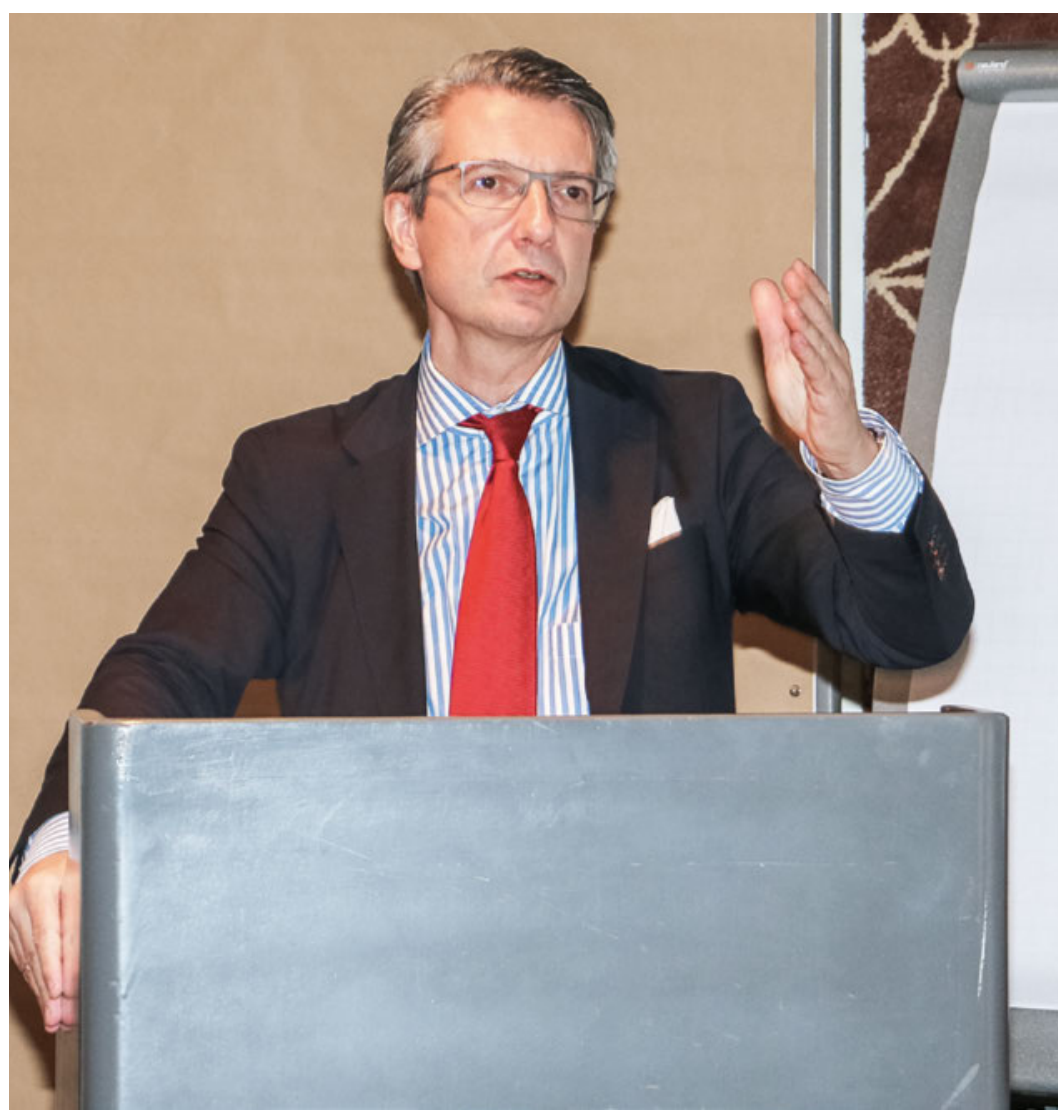

Willy Oggier intervenant, le juriste Rainer Hess, s'est montré particulièrement efficace. Directeur général de l'Union fédérale des médecins conventionnés en Allemagne jusqu'en 2005, il a ensuite repris la présidence de la Commission fédérale commune, dont les organisations responsables sont les associations faîtières des caisses-maladie obligatoires, l'Union fédérale des médecins conventionnés, l'Union fédérale des dentistes conventionnés et la Société allemande des hôpitaux. Dans le cadre de ces fonctions, il a acquis de précieuses expériences en matière de budgets globaux.

\section{Une politique des dépenses orientée sur les recettes}

R. Hess a précisé d'emblée que le système de santé allemand ne connaît pas un budget global au sens d'une «limite des dépenses fixes englobant tous les domaines de soins». Une démarche législative dans ce sens a échoué au Conseil fédéral allemand, la Chambre des Länder. Le "principe de la stabilité du taux de contribution» a en revanche été maintenu. Comme l'a expliqué l'économiste Volker Ulrich, on entend par là une "politique des dépenses orientée sur les recettes», qui se caractérise par le fait que «seule la somme d'argent correspondant à celle de l'année précédente peut être dépensée dans un domaine de dépenses déterminé, par année civile, pour l'ensemble des assurés de l'assurance obligatoire; cette somme peut être adaptée en fonction du pourcentage de l'augmentation de la masse salariale de base». Le but est d'éviter que la part de salaire nécessaire au financement de l'assurance-maladie ne connaisse une croissance disproportionnée.

Pour les politiciens, cette idée peut sembler attrayante. Mais comme l'ont expliqué les deux orateurs allemands, R. Hess et V. Ulrich, sa mise en œuvre s'accom- 


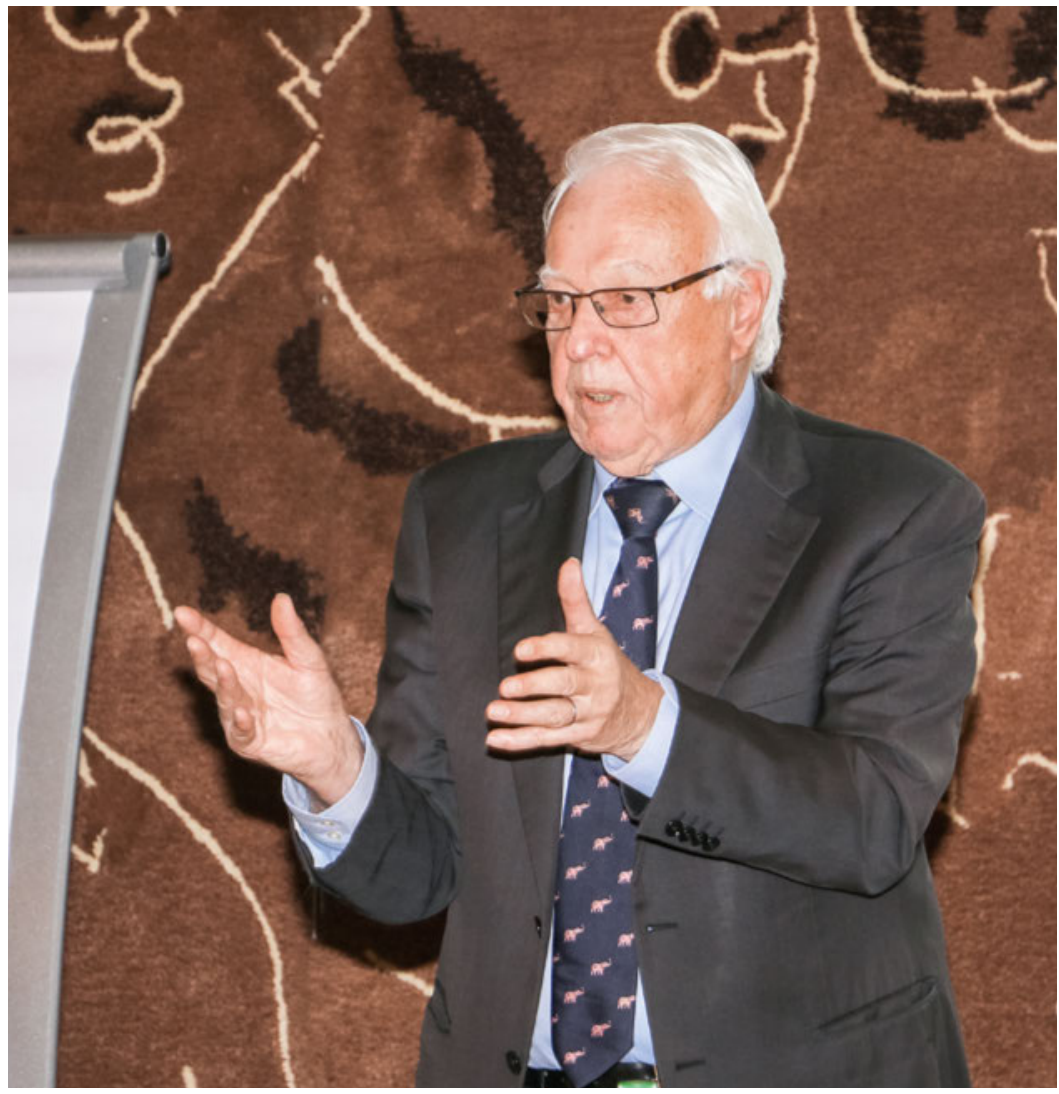

Rainer Hess

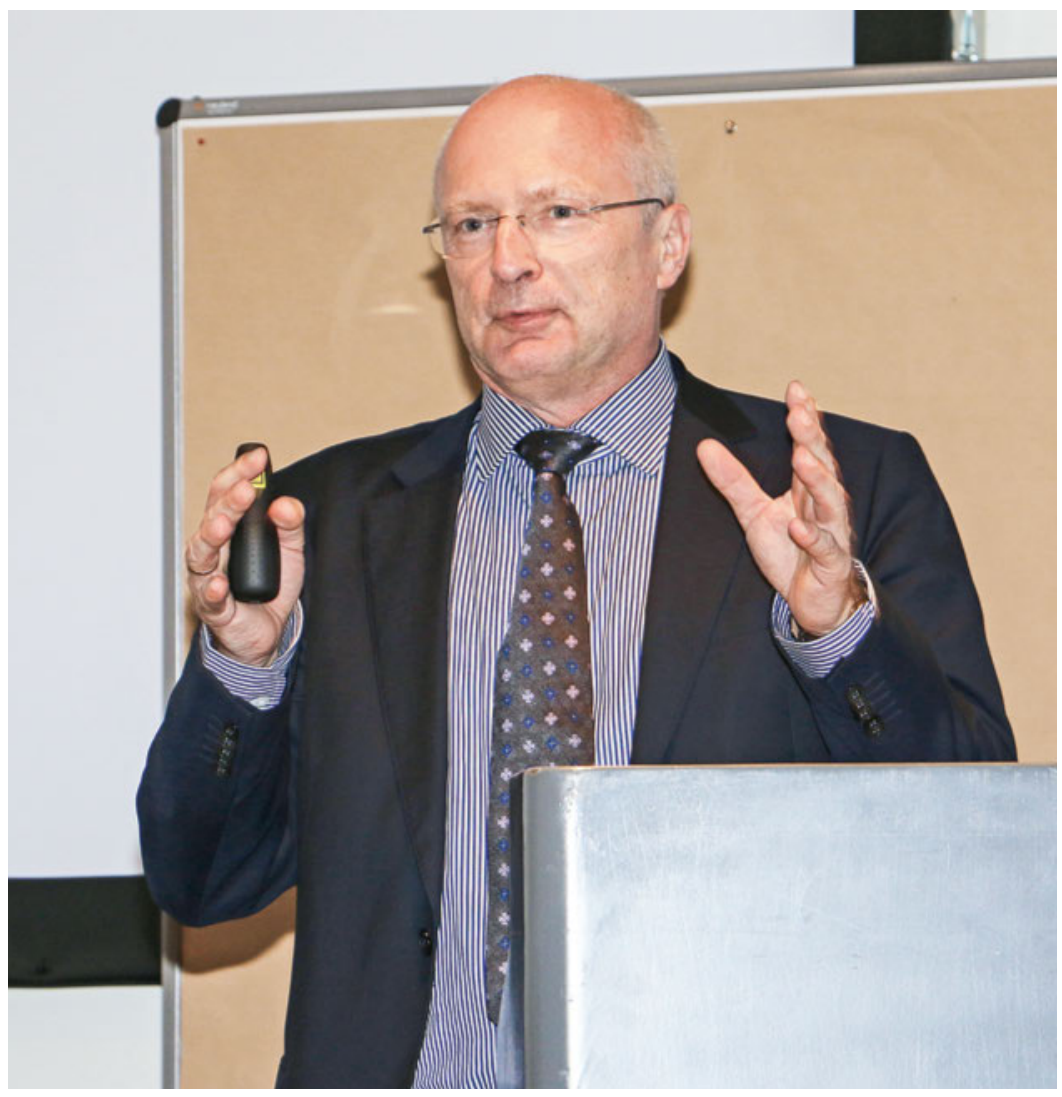

Volker Ulrich pagne de divers problèmes. Ces derniers requièrent des mécanismes correctifs qui s'écartent considérablement de la simplicité du modèle initial. Rainer Hess s'est aussi montré surpris de l'intérêt de la Suisse mais pour une autre raison. En Allemagne, l'assurance-maladie obligatoire fonctionne selon le principe des prestations en nature, alors que la Suisse applique le principe du remboursement des coûts. Cela signifie qu'en Allemagne, les médecins conventionnés fournissent leurs prestations sans avoir droit au versement d'un montant fixe pour la prestation fournie. Ils ne peuvent prétendre qu'à une participation à la répartition des honoraires. Pour R. Hess, il est tout à fait possible d'établir des budgets dans ces conditions, alors qu'en Suisse, il faudrait entièrement changer le système, chaque prestation médicale étant rémunérée par un montant fixe.

\section{Bilan négatif des experts allemands}

Le problème principal du système allemand réside dans le fait que le montant de la rémunération totale (du «budget global») n'est pas influencé par des facteurs tels que l'évolution démographique, l'évolution de la morbidité, le progrès médical ou l'évolution du nombre de médecins. Seule l'augmentation de la somme salariale de base, c'est-à-dire un paramètre extérieur, est déterminante. Le bilan des experts allemands appuie nettement sur les inconvénients par rapport aux avantages des budgets globaux. Pour R. Hess et V. Ulrich, les avantages se limitent au fait que les budgets globaux constituent effectivement un moyen de fixer une limite supérieure des dépenses dans l'assurance obligatoire. Les problèmes commencent déjà lorsqu'il s'agit de définir les critères sur la base desquels fixer un budget global. Selon lui, une chose est sûre, le médecin qui réussit à traiter le moins de personnes malades est celui qui est le plus efficient dans un budget global. Etant donné que le budget du moins en Allemagne - ne s'oriente pas selon les besoins de traitement, les coupes dans les soins sont inévitables. Le rationnement des prestations médicales augmente, ce qui engendre une médecine à deux vitesses avec des listes d'attente et une sélection des patients.

\section{Le budget global dans l'assurance, un corps étranger}

Le juriste saint-gallois Ulrich Kieser a présenté un aperçu juridique du thème du budget global et rappelé que «l'assurance se caractérise par la prestation garantie en cas de survenance de l'évènement redouté». Le 


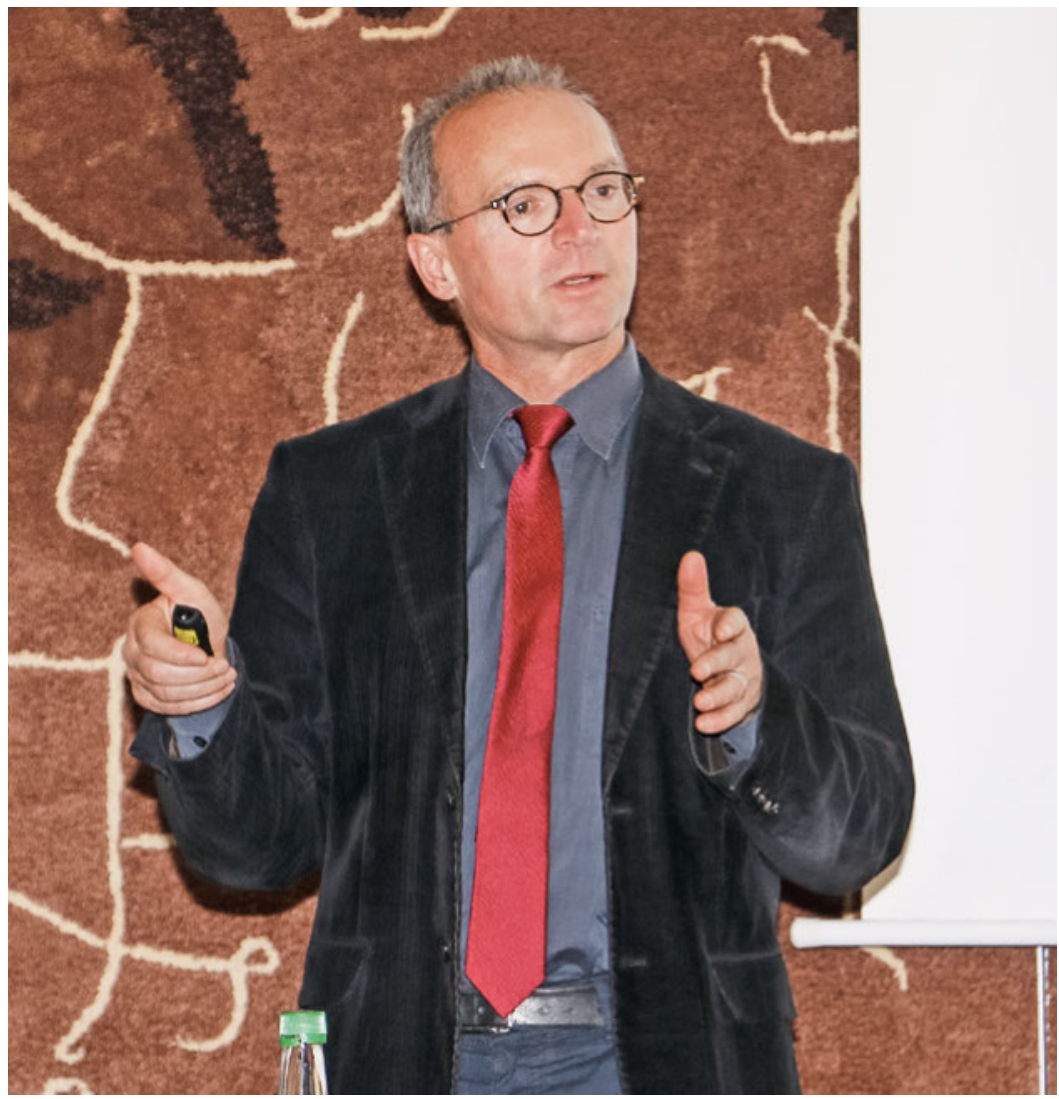

Ulrich Kieser

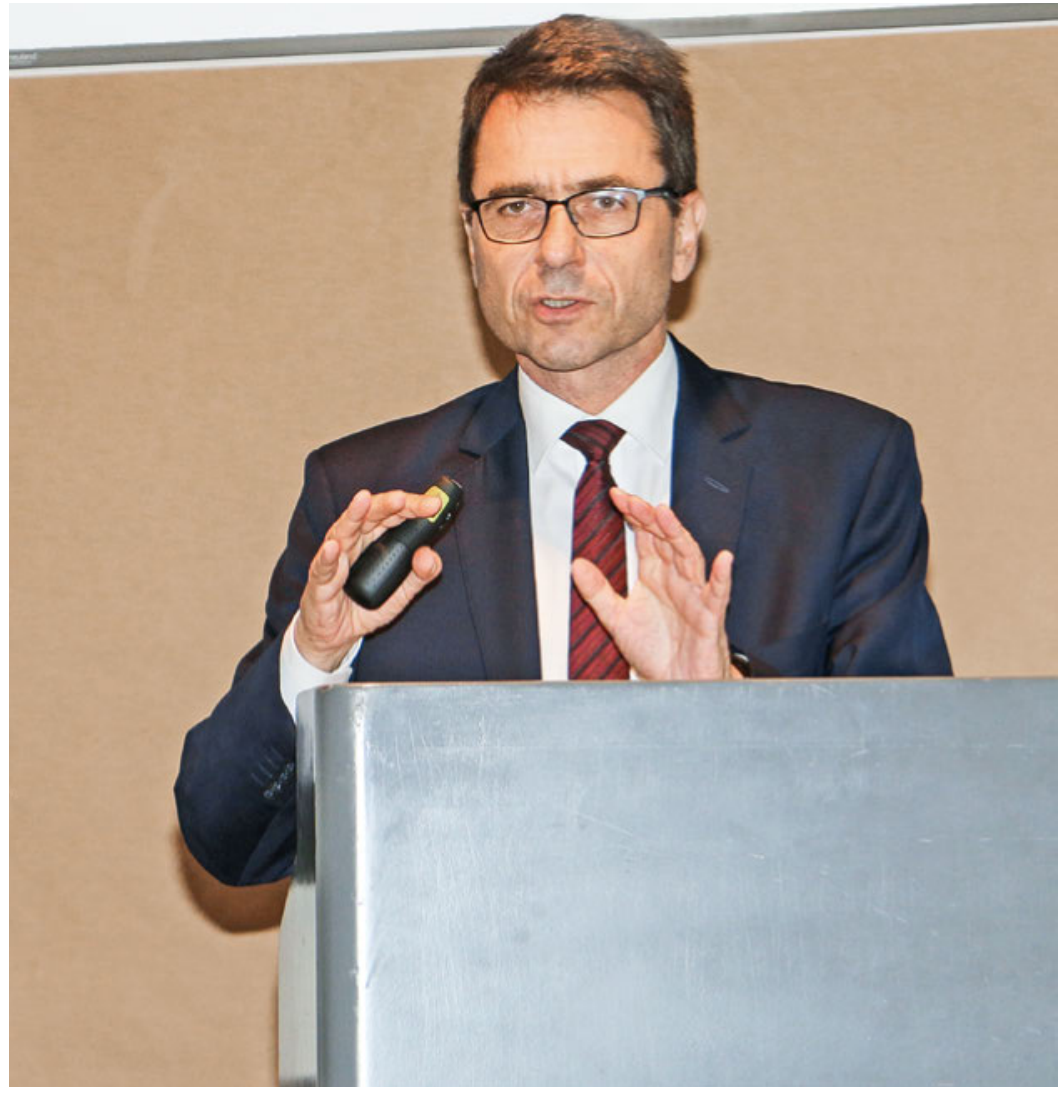

Oliver Peters besoin de financement est couvert par une "contrepartie», généralement sous forme de prime. Il a aussi rappelé que la Loi fédérale sur l'assurance-maladie (LAMal) précise que les soins doivent être appropriés et leur qualité de haut niveau, tout en étant le plus avantageux possible. La question du montant total des coûts est d'une certaine manière secondaire, a poursuivi U. Kieser. Un budget global ne peut pas limiter le droit à la prestation et représente donc un «corps étranger» dans l'assurance. U. Kieser a aussi souligné qu'aucune branche dans l'assurance sociale suisse (AVS, AI, assurance-accidents, prévoyance professionnelle) n'applique un budget global. Il s'est rallié à l'avis des orateurs précédents: le rattachement d'un budget global à une valeur telle que l'évolution des salaires nominaux est inapproprié. U. Kieser a également rappelé que l'atteinte de la limite du budget global soulève de délicats problèmes juridiques. En effet, comme il n'est pas possible de limiter le droit aux prestations, une correction devrait s'effectuer par le biais de la rémunération des fournisseurs de prestations. Ce faisant, il faudrait tenir compte du principe de l'égalité de traitement et de l'interdiction de l'arbitraire. D'après U. Kieser, il n'est pas admissible d'obliger des fournisseurs de prestations n'ayant pas auparavant fourni des prestations excessives à fournir les mêmes prestations à un tarif ou à un prix inférieur.

Pour conclure, U. Kieser a constaté qu'un budget global minerait le système d'assurance actuel et qu'il faudrait par conséquent porter un regard critique, du point de vue constitutionnel, sur l'introduction générale d'un tel système. Sans oublier qu'un budget global rigide s'accompagnerait d'épineuses questions de mise en œuvre.

\section{L'expérience des cantons de Vaud et du Tessin}

Oliver Peters, directeur général adjoint au CHUV à Lausanne, et Christian Camponovo, directeur de la Clinica Luganese à Lugano, ont parlé des expériences faites dans des cantons qui travaillent déjà avec des budgets globaux dans le domaine des soins hospitaliers. Dans leurs cantons, un budget global est négocié pour chaque institution. Dans son exposé, Ulrich Kieser avait déjà rendu attentif au fait que les hôpitaux et établissements médico-sociaux sont pilotés selon les besoins, ce qui facilite et rend possible d'établir un budget réaliste, tout en ajoutant que ce procédé ne peut pas simplement être transposé dans le domaine ambulatoire. Fin 2006, le canton de Vaud a d'ailleurs supprimé les budgets globaux qui couvraient les domaines hospitalier et ambulatoire. Depuis 2007, les budgets 


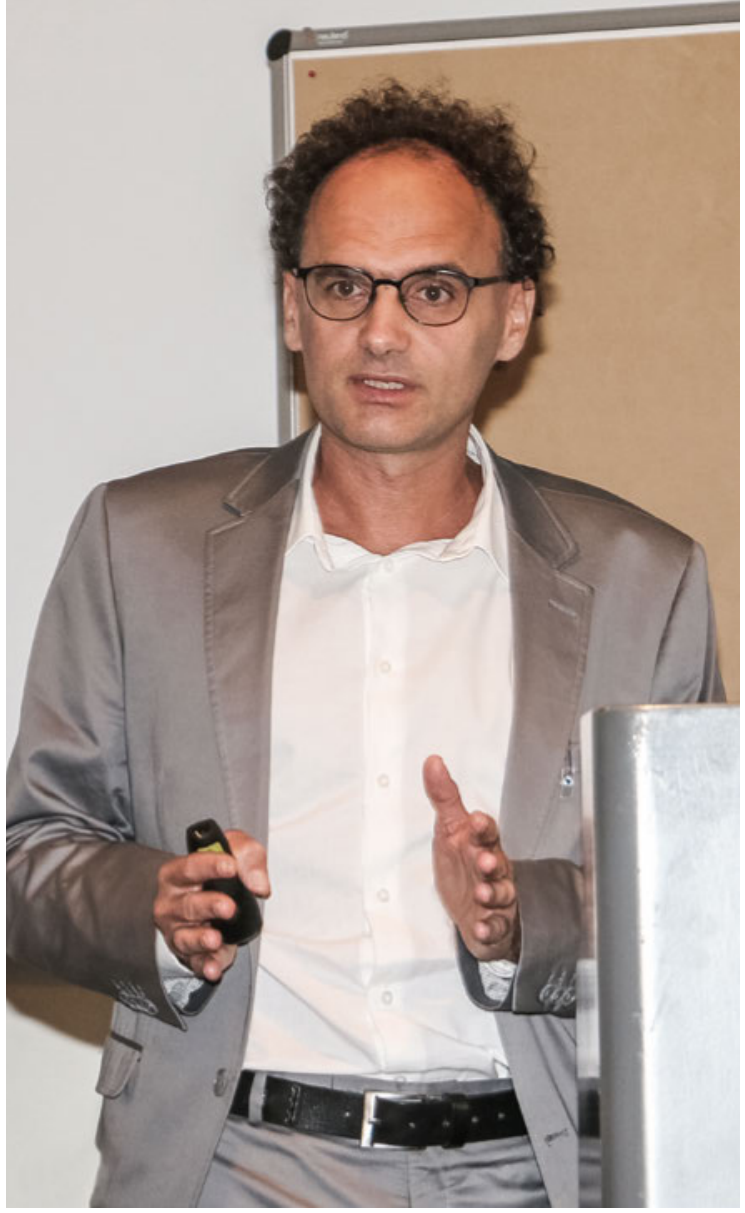

Christian Camponovo

globaux ne concernent plus que les prestations hospitalières.

Les deux gestionnaires d'hôpitaux ont tiré des bilans très différents. Du point de vue d'Oliver Peters, un budget global est un outil utile pour contrôler l'augmenta- tion du volume. Pour être efficace, il doit cependant être aménagé de façon globale et différenciée. Quant aux effets de rationnement, ils peuvent être évités par une correction des prestations et une obligation d'admission. Christian Camponovo a conclu que le bilan positif tiré par son collègue du CHUV s'expliquait probablement du fait que, par rapport à une clinique privée comme celle qu'il dirige, un hôpital universitaire dispose de meilleures cartes pour négocier un budget. Quoi qu'il en soit, il n'a pas partagé le point de vue d'Oliver Peters. D'après son expérience, les budgets globaux ne résolvent pas - ou résolvent seulement en apparence-les problèmes visés. Dans le meilleur des cas, les coûts ne baissent qu'à brève échéance et un effet positif sur les taux d'hospitalisation n'est pas perceptible. En revanche, les budgets globaux amènent de nombreux problèmes pratiques pour les patients et les fournisseurs de prestations, notamment du fait qu'ils génèrent de fausses incitations. Parmi les exemples de répercussions négatives, Christian Camponovo a cité une concurrence réduite, une baisse des investissements dans la qualité et des difficultés budgétaires lorsqu'il s'agit d'engager rapidement de la maind'œuvre hautement qualifiée.

\section{En fin de compte, le scepticisme règne}

Dans leur grande majorité, les experts intervenant lors du séminaire n'ont guère trouvé d'avantages à l'application de budgets globaux à large échelle. Ils n'y voient pas une solution pour résoudre les problèmes de financement du système de santé suisse. Reste à voir si la politique parviendra à des conclusions similaires.

\section{Crédit photos \\ Bruno Kesseli}

